\title{
Growth of an Ordered Crystalline Organic Heterojunction**
}

\author{
By Richard R. Lunt, Jay B. Benziger, and Stephen R. Forrest*
}

Organic semiconductors have gained tremendous attention over the last two decades due to their potential for use in thin film transistors (TFTs), sensors, solar cells and organic light emitting diodes: ${ }^{[1]}$ The best device performances (particularly for TFTs) have often been achieved using free standing single crystals. ${ }^{[2-4]}$ However, in general the size, thickness, and shape of such crystals are not easily controlled. ${ }^{[5]}$ Many researchers have therefore focused on methods for producing highly aligned crystalline organic thin films through substrate templating. ${ }^{[6,7]}$ In this work, we demonstrate the quasi-epitaxial growth of an ordered heterojunction between two archetypal organic molecular layers consisting of copper phthalocyanine (CuPc) and 3,4,9,10-perylenetetracarboxylic dianhydride (PTCDA) on a single crystal $\mathrm{KBr}$ substrate using organic vapor phase deposition (OVPD). The surface mesh for single layer growths of PTCDA and $\mathrm{CuPc}$, monitored in situ and in real time by high pressure reflection high energy electron diffraction (HP-RHEED), were found to orient over extended substrate distances $(\sim 2 \mathrm{~cm})$ along the [100] and [110] $\mathrm{KBr}$ lattice directions, respectively, for film growth of up to several hundred Ångstroms thick. Furthermore, highly ordered PTCDA films are shown to be a suitable surface for subsequent growth of ordered films of CuPc.

The two archetypal molecules, PTCDA and CuPc, have both been proven useful in organic electronic applications. Also, both have been quasi-epitaxially ${ }^{[8]}$ grown on weakly binding metal surfaces, ${ }^{[9-11]}$ and on highly oriented pyrolytic graphite (HOPG). ${ }^{[12-14]}$ Growth on alkali-halide $(\mathrm{AH})^{[15-17]}$ substrates with a clear relationship between film and substrate has been used to achieve epitaxial growth, although ordered films of only a few monolayers (ML) thickness could be grown prior to structural collapse due to large accumulated

[*] Prof. S. R. Forrest, R. R. Lunt Departments of Electrical Engineering \& Computer Science Physics, and Materials Science \& Engineering University of Michigan

Ann Arbor, Michigan 48109 (USA)

E-mail: stevefor@umich.edu

R. R. Lunt, Prof. J. B. Benziger

Department of Chemical Engineering

Princeton Institute for the Science and Technology of Materials (PRISM)

Princeton University

Princeton, NJ 08544 (USA)

[**] The authors gratefully acknowledge the Air Force Office of Scientific Research, Universal Display Corp. and Global Photonic Energy Corp. for partial support of this work. strain energies. Additionally, the organic-organic heteroepitaxy of MLs of PTCDA grown on MLs of other organic molecules has been demonstrated. ${ }^{[18,19]}$ While many of these studies have revealed the fundamental differences between the forms of organic and inorganic epitaxy, nearly all have been limited to growth in ultrahigh vacuum, and involve only a few MLs, neglecting the issues of structural evolution into more practically useful thicker films. The few reports of quasi-epitaxial evolution of thick films have been limited to growth conditions where discontinuous, strain-relieved 3D islanding occurs after the formation of an ultrathin (1-2 ML) oriented wetting layer, following the Stranski-Krastanow growth mode. ${ }^{[17,20-22]}$ Moreover, we are unaware of demonstrations of long range azimuthal order greater than the microscopic image field size of $(1 \mathrm{~nm}-1 \mu \mathrm{m})^{2}$ for several hundred Ångstrom thick film growth. For example, although PTCDA has been grown on a number of $\mathrm{AH}$ substrates including $\mathrm{NaCl}, \mathrm{KCl}$, $\mathrm{KI},{ }^{[17]}$ and $\mathrm{KBr},{ }^{[21,23]}$ to our knowledge, no evidence of quasiepitaxial alignment has been reported for growth on $\mathrm{KBr}$.

The HP-RHEED pattern for the initial $\mathrm{KBr}$ substrate is shown in Figure 1a, indicating a flat and ordered surface for growth. Subsequent HP-RHEED patterns for $400 \AA$ thick films of PTCDA grown at a substrate temperature of $T_{\mathrm{g}}=-15^{\circ} \mathrm{C}$ (see Experimental Sec.) are shown in Figure 1 for a $0.1 \times 20 \mathrm{~mm}^{2}$ incident beam parallel to the [100] (see Fig. 1b), and [110] (Fig. 1c) KBr crystal directions. Scanning electron microscopy (SEM) images of the corresponding PTCDA surface are shown for $T_{\mathrm{g}}=-15^{\circ} \mathrm{C}$ and $75^{\circ} \mathrm{C}$ (Fig. $1 \mathrm{~d}$ and e). At both temperatures, PTCDA films were found to stack in the [102] direction of the $\alpha$-phase (see Fig. 2), as determined from X-ray data.

In the [100] direction, two peaks are assigned to the (20) and the (02) surface mesh directions of the flat-lying PTCDA (see Fig. 1b). This index pair results from the 4-fold symmetry of the $\mathrm{KBr}$ substrate, where a rotation of $90^{\circ}$ is energetically equivalent for the PTCDA unit cell alignment. Along the [110] direction, several orders of $(n n)$ and $(n 2 n)$ are observed, with no evidence for the $(0 n)$ or $(n 0)$. This suggests that while there are equivalent, $90^{\circ}$ rotated domains, all domains align either along [100] or [010]. This symmetry also explains, for example, why both the $(n n)$ and $(n 2 n)$ appear simultaneously, where a rotation of the surface mesh by $90^{\circ}$ results in a near overlap, in angle, of these two reciprocal planes. Several orders of the $(n 4 n)$ are also observed, but are difficult to distinguish, for example, from the $(2 n 3 n)$. There is negligible change in the fitted lattice parameters for switching the (1 4) index to (2 3); the former is slightly more consistent with the $d$-spacings where $d_{\text {meas }}=4.7 \pm 0.1 \AA, d_{14}=4.62 \pm 0.01 \AA$, 

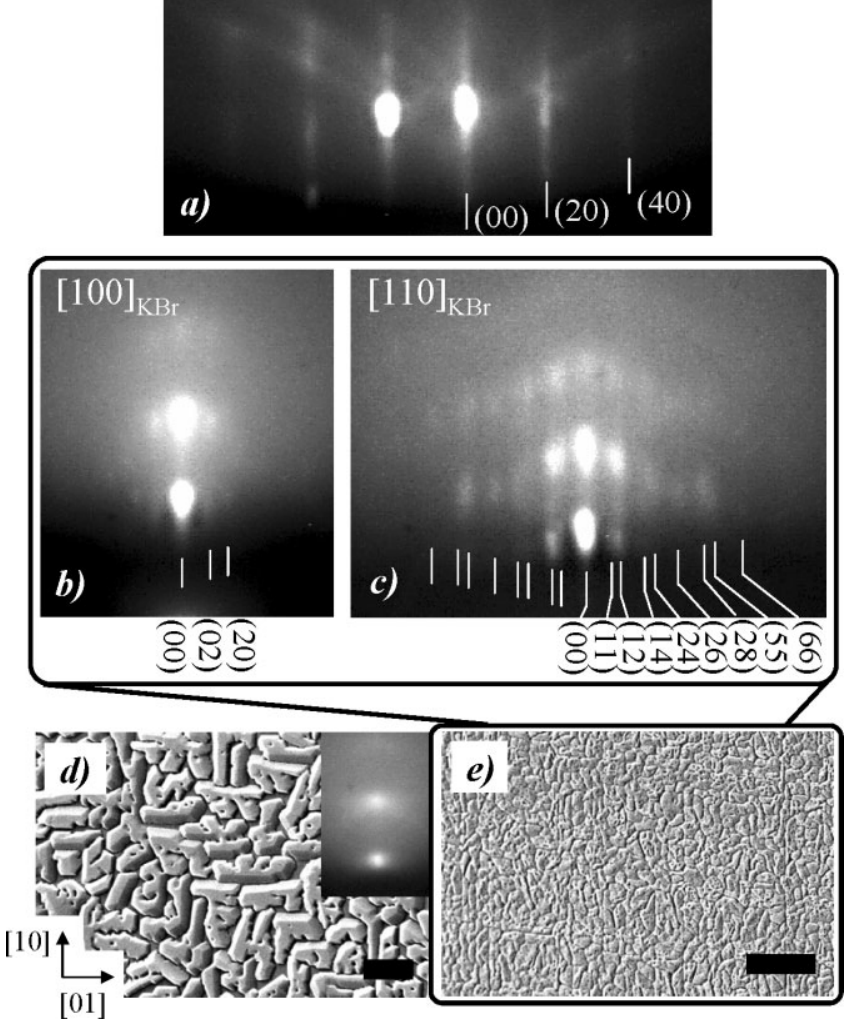

Figure 1. a) Reflection high energy electron diffraction (RHEED) pattern for a bare $\mathrm{KBr}$ substrate with the electron beam oriented in the [100] direction. RHEED patterns for $400 \AA$ of PTCDA grown on $\mathrm{KBr}$ at $T_{g}=-15{ }^{\circ} \mathrm{C}$ with a beam oriented approximately along the (a) [100] and (b) [110] directions of the $\mathrm{KBr}$ substrate. Streak locations are highlighted with white tick marks. The measured d-spacings are (a) (02), $(20)=9.7 \AA, 6.0 \AA$, respectively, and (b) (11), (12), (14), (24), (26), (28), (55), $(66)=10.4 \AA, 7.9 \AA, 4.7 \AA, 3.9 \AA$, $2.96 \AA, 2.29 \AA, 2.10 \AA, 1.72 \AA$, respectively. (d,e) Scanning electron microscope (SEM) images of $400 \AA$ of PTCDA grown on $\mathrm{KBr}$ at (d) $T_{\mathrm{g}}=75^{\circ} \mathrm{C}$ and (e) $T_{\mathrm{g}}=-15^{\circ} \mathrm{C}$ and a growth pressure of 60 mTorr. The black scale bar in both (d) and (e) corresponds to $1 \mu \mathrm{m}$. From atomic force microscopy, the average roughnesses and feature heights are $210 \AA, 500 \AA$ for (d) and $35 \AA, 90 \AA$ for (e). Inset of (d) is a characteristic RHEED pattern for (d). The $\mathrm{KBr}$ substrate orientation for both (d) and (e) is indicated in (d); all subsequent SEM images have similarly oriented substrates. The highlighted box around (b, c, and e) indicate the data were taken for the same growth.

$d_{23}=4.50 \pm 0.01 \AA$, but the latter can be observed alongside the $(n n)$ and $(n 2 n)$ without grain misalignment. Identification of the two orientations correspond to that observed at all orientations for less aligned monolayers of PTCDA on $\mathrm{Au}(111)$, where the (01), (11), (12), (20), (14), (24), (40), (55), (66) etc. were all simultaneously observed. ${ }^{[11]}$

The regression analysis of all the $d$-spacings yielded a PTCDA surface mesh of $b_{1}=19.9 \pm 0.2 \AA$ and $b_{2}=12.2 \pm 0.1 \AA$, which is close to the bulk surface mesh of the $\alpha$-phase oriented in the [102] direction, where $b_{1}=19.91 \AA$ and $b_{2}=11.96 \AA .{ }^{[6]}$ HP-RHEED patterns recorded during growth indicated that the lattice did not vary significantly with thickness (within measurement error). The orientation of the PTCDA with the $\mathrm{KBr}$ substrate, deduced
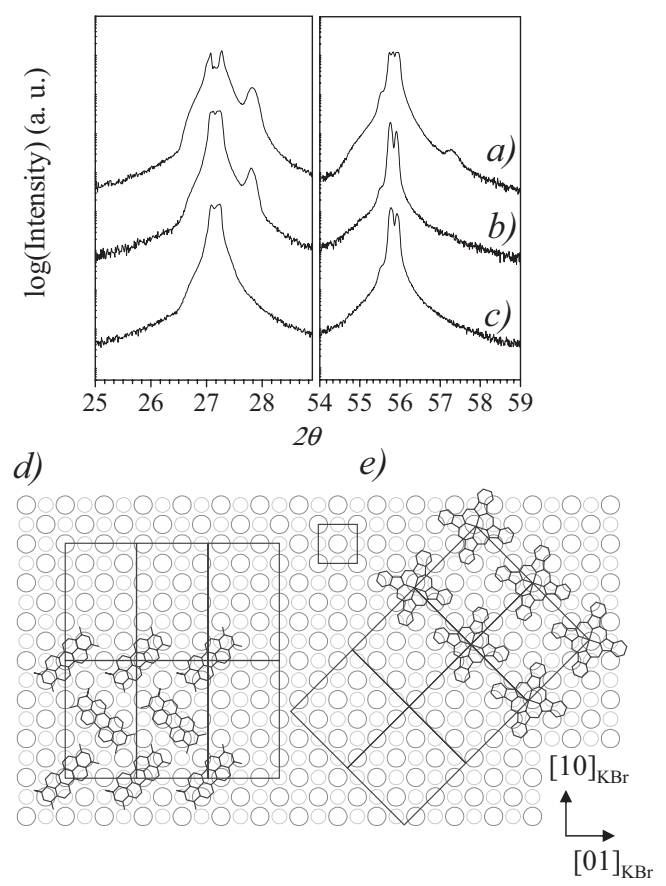

Figure 2. X-ray $\mathrm{Cu} \mathrm{K} \alpha \theta-2 \theta$ diffraction scans for a) PTCDA on $\mathrm{KBr}$ b) CuPc on $\mathrm{KBr}$, and c) bare $\mathrm{KBr}$. Both the (102) and (204) PTCDA diffraction peaks at $\left(2 \theta=27.80^{\circ}\right.$ and $\left.57.28^{\circ}\right)$, can be observed corresponding to a $d$-spacing of $3.21 \AA$, helping to distinguish between the $\alpha$-phase $(d=3.20 \AA)$ and the $\beta$-phase $\left(d=3.25 \AA, 2 \theta=27.41^{\circ}\right)$. The X-ray patterns were found to be independent of the growth conditions. d) and e) Model of the overlayer alignment for (d) PTCDA and (e) CuPc on KBr. The potassium ions are slightly smaller than the bromine ions. $\mathrm{The} \mathrm{KBr}$ unit cell is also indicated. The molecular alignment is assumed from the bulk phase crystal structure for PTCDA and from Ref. [10] for CuPc.

from the HP-RHEED patterns and streak indexing, is shown schematically (to within $\pm 5^{\circ}$ ) in Figure 2d. While the unit cell is incommensurate, a $6 \times 1$ supercell is nearly commensurate. The measured strain for a $6 \times 1$ PTCDA superstructure was measured to be $(0.7 \pm 0.7 \%, 0.8 \pm 0.6 \%)$ in the $\left(b_{1}, b_{2}\right)$ directions, where the measured superstructure lattice strain is slightly reduced from the bulk phase lattice.

From the SEM images, it is clear that the PTCDA film grown at $T_{\mathrm{g}}=-15^{\circ} \mathrm{C}$ (Fig. 1e), has reduced surface roughness and smaller domains than the film grown at $T_{\mathrm{g}}=75^{\circ} \mathrm{C}$ (see Fig. 1d) which is confirmed with atomic force microscopy (AFM). Despite the apparent formation of crystalline domains in Figure 1d, the HP-RHEED patterns obtained at $T_{\mathrm{g}}$ $>75{ }^{\circ} \mathrm{C}$ (inset Fig. 1d) are spotty and diffuse, with very little azimuthal orientation, indicative of a lack of in-plane order. This counterintuitive trend has been observed for PTCDA previously on other substrates, ${ }^{[6]}$ and could be due to a highly mobile, liquid-like film formation of the first deposited monolayer which traps in-plane disorder as subsequent deposited molecules arrive and induce crystallization.

In contrast, the growth of $\mathrm{CuPc}$ on $\mathrm{KBr}$ showed improved alignment at higher temperatures, similar to growth on other substrates. ${ }^{[2]}$ At $T_{\mathrm{g}}=65^{\circ} \mathrm{C}$, a uniform orientational dependence across the substrate was observed in Figure $3 a$ and b, 


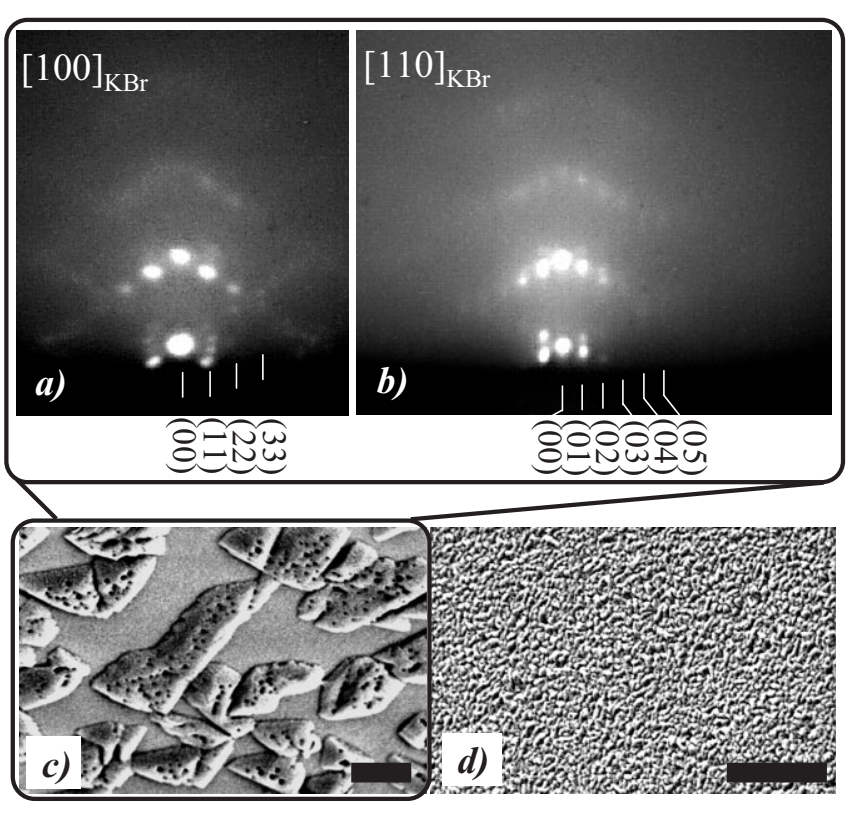

Figure 3. RHEED patterns for the growth of $700 \AA$ (nominal) of CuPc at $T_{\mathrm{g}}=65{ }^{\circ} \mathrm{C}$ are shown for a beam alignment of a) [100] and b) [110] direction of the $\mathrm{KBr}$ substrate. RHEED patterns are on the same scale of those in Figure 1a-c. Spot locations are highlighted with white tick marks. The measured $d$-spacings are a) (11), (22), (33) $=10.0 \AA, 5.0 \AA, 3.33 \AA$, re spectively, and b) (01), (02), (03), (04), (05) = 14.0 $\mathrm{A}, 6.8 \AA, 4.6 \AA$, $3.41 \AA$, $2.71 \AA$, respectively. SEM images of $700 \AA$ CuPc thick film grown on $\mathrm{KBr}$ at c) $T_{\mathrm{g}}=65^{\circ} \mathrm{C}$ and d) $T_{\mathrm{g}}=0{ }^{\circ} \mathrm{C}$ and at a growth pressure of 10 mTorr. The black scale bar in both (c) and (d) correspond to $1 \mu \mathrm{m}$. From AFM, the average roughnesses and feature heights are $550 \AA$, $1800 \AA$ for (c) and $50 \AA$, $105 \AA$ for (d). Incomplete 3D coverage is apparent in (c) whereas (d) exhibits a rough but continuous film morphology. The highlighted box around $(a-c)$ indicate the data are taken for the same growth.

despite the formation of large 3D islands apparent in Figure $3 c$. Many orders of $(n n)$ and $(0 n)$ are observed along the [100] and [110] $\mathrm{KBr}$ directions (Fig. 3a), respectively, for $T_{\mathrm{g}}=65^{\circ} \mathrm{C}$ indicating either a single preferred alignment for the 3-D crystallites, or a nearly square lattice. At $T_{\mathrm{g}}=0{ }^{\circ} \mathrm{C}$, the HP-RHEED patterns indicate a weaker orientational dependence, with the appearance of additional streaks belonging to neither $(n n)$ nor $(0 n)$, although the film surface flatness improved (see Fig. 3d).

A single x-ray peak at $2 \theta=27.8^{\circ}(d=3.20 \AA)$ seen in Figure $2 \mathrm{~b}$ indicates that the CuPc molecules lie flat on the $\mathrm{KBr}$ substrate. The $d$-spacings measured from both the HPRHEED patterns at the different azimuthal angles, and from $\mathrm{x}$-ray diffraction, do not coincide with the $d$-spacings for any of the commonly observed bulk phases $(\alpha$ or $\beta)$. For example, the surface mesh of the $a$-phase stacked in the [313] direction is expected to be $b_{1}=26.49 \AA, b_{2}=14.15 \AA, \beta=100.3^{\circ}$, $d_{h k l}=3.18 \AA$. Instead the measured $d$-spacings match with the surface mesh for one of the phases found on $\operatorname{Ag}(111) .^{[10]}$ Using the surface mesh parameters for this phase to index the pattern, we infer a lattice mesh of $b_{1}=14.7 \pm 0.2 \AA$, $b_{2}=13.6 \pm 0.1 \AA$, and $\beta=90 \pm 5^{\circ}$ from HP-RHEED. Note that a square CuPc lattice of $(14.0 \AA)^{2}$ oriented along the [110] substrate direction would be perfectly commensurate. Instead, the observed lattice is only approximately commensurate over a large $6 \times 1$ superlattice, resulting in a significant superstructure strain of $(0 \pm 1 \%, 3.0 \pm 0.5 \%)$ in the $\left(b_{1}, b_{2}\right)$ directions. The high degree of strain along $b_{2}$ may provide the driving force for the 3D morphology at higher growth temperatures that is relieved through a stacking distortion similar to tetragonal distortion seen in cubic lattices. The alignment of the CuPc surface mesh is oriented along [110] of $\mathrm{KBr}$ as shown in Figure 2e. This orientation is in contrast to that found for $\mathrm{CuPc}$ grown by OMBD, where the lattice was weakly aligned over a $12^{\circ}$ range along [100] and [010]. ${ }^{[15]}$ The reason for the discrepancy is unclear, but may be related to the diffusive growth characteristics of OVPD which can impact the kinetic energy of arriving molecules.

By growing at low substrate temperature, surface diffusion is suppressed and the nucleation rate increases, resulting in full substrate coverage. However, to achieve ordered films under these conditions, there must be a mechanism that drives short-range molecular rearrangement. For example, it has been shown that flat lying PTCDA has a rotational degree of freedom around its central benzyl ring. ${ }^{[25]}$ This might allow for subtle relaxations into preferred alignments without requiring surface diffusion beyond the range of a few molecules. On the other hand, $\mathrm{CuPc}$ prefers to crystallize in an out-of-plane herringbone structure which requires additional energy to fully orient to the substrate lattice. The low pressure environment typical of OVPD can also play a role in increasing molecular surface mobility, where collisions with the nitrogen carrier gas can enhance surface site sampling without the need for substantial molecular diffusion.

Growth of CuPc on $400 \AA$ thick films of PTCDA (under the same conditions as in Fig. 1b, c, and e for PTCDA and Fig. 3d for $\mathrm{CuPc}$ ), were found to form ordered heterostructures. The HP-RHEED patterns for this multilayer growth are shown in Figure $4 \mathrm{a}$ and $\mathrm{b}$, and were indexed using the surface mesh of the $\mathrm{CuPc}$ grown directly on $\mathrm{KBr}$. The pattern can be qualitatively described as a superposition of the patterns in Figure 3a and $b$. While this superposition implies that there are two distinct domains rotated by $45^{\circ}$, this same pattern was observed over a range of azimuthal angles. This lack of azimuthal variation implies that there is no strict alignment of the CuPc lattice to the underlying PTCDA layers despite a quasi-epitaxial relationship between the PTCDA and $\mathrm{KBr}$ lattices. Further, the degree of in-plane ordering is similar to that of $\mathrm{CuPc}$ grown on $\mathrm{HOPG}$, as reported previously where $\mathrm{CuPc}$ was found was found to weakly align with the HOPG lattice. ${ }^{[24]}$ It was also found that the growth temperature did not strongly affect the alignment here, but instead impacted the morphology and wetting of the CuPc films. This is apparent in Figure $4 \mathrm{c}$ and $\mathrm{d}$, and is similar to the growth of CuPc directly on $\mathrm{KBr}$. A comparison of Figures $3 \mathrm{~d}$ and $4 \mathrm{~d}$ indicates that $\mathrm{CuPc}$ replicates the underlying morphology of the PTCDA. In contrast, when a second layer of PTCDA was grown on the surface of the $\mathrm{CuPc} / \mathrm{PTCDA} / \mathrm{KBr}$ structure, the streaks in the 


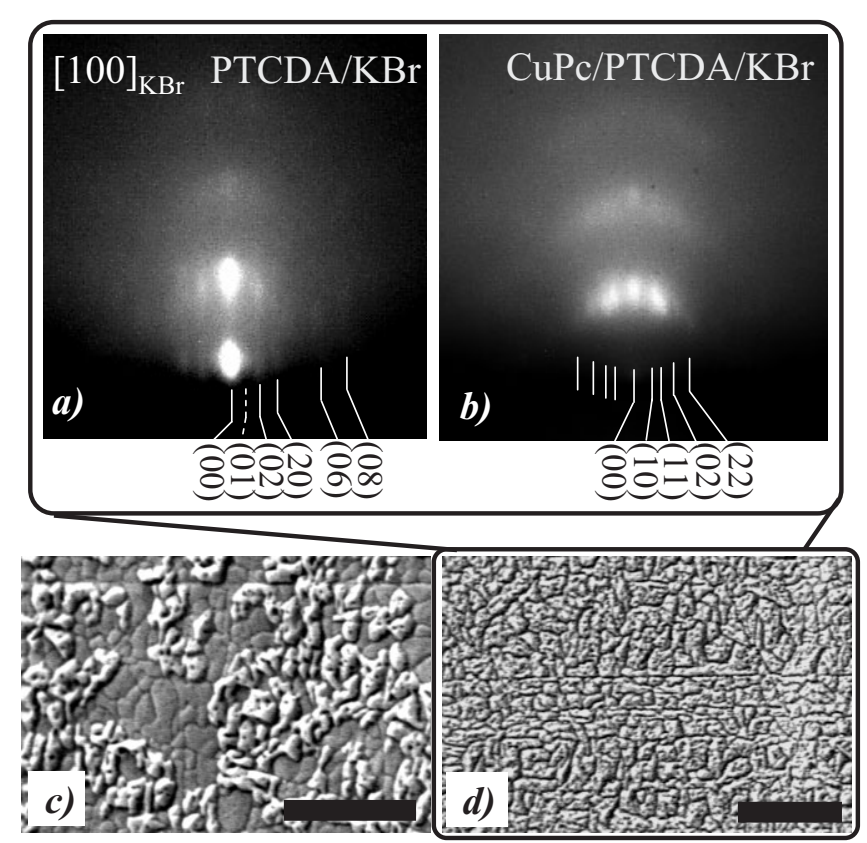

Figure 4. RHEED patterns of both (a) the initial $400 \AA$ thick PTCDA growth surface oriented along the [100] direction (grown under similar conditions to Fig. 1a, b, and e), and (b) the subsequent growth of $150 \AA$ thick films of CuPc on this PTCDA surface. Streak locations are highlighted with white tick marks. The measured $d$-spacings are a) (01), (02), (20), (06), (08) = 20.4 $\AA$, $9.9 \AA, 6.2 \AA, 3.20 \AA, 2.47 \AA$, respectively, and b) (10), (11), (02), (22) = 14.5 $\mathrm{A}, 10.1 \AA, 6.9 \AA$, $5.0 \AA$, respectively. Image (b) was observed for all azimuthal rotations. SEM images of $150 \AA$ thick films of CuPc grown on PTCDA at (a) $T_{g}=90^{\circ} \mathrm{C}$ and (b) $T_{g}=0{ }^{\circ} \mathrm{C}$. The black scale bar in both (c) and (d) correspond to $1 \mu \mathrm{m}$. From AFM, the average roughnesses and feature heights are $180 \AA$, $500 \AA$ (top of PTCDA to top of CuPc features) for (c) and $70 \AA$, $160 \AA$ for (d). The underlying PTCDA can be observed in (c) due to the difference in contrast between the CuPc and PTCDA, which is likely due to the differing metal content.

HP-RHEED patterns degenerated into rings, indicating a nearly complete loss of in-plane order. We therefore conclude that while PTCDA provides a growth template for $\mathrm{CuPc}$, the converse is not true. This observation is consistent with other reports, ${ }^{[12]}$ and may be due to the non-planar molecular packing commonly observed for CuPc or possibly due to a higher surface energy for $\mathrm{CuPc}$ than PTCDA. Since the accurate structural refinement of this $\mathrm{CuPc}$ phase is unknown, the surface free energy cannot be accurately calculated for such a comparison.

In summary, we have demonstrated the OVPD growth of both PTCDA and CuPc thick films on an alkali halide substrate with long range crystalline order. Furthermore, we have demonstrated a highly ordered heterojunction consisting of bulk thin films of these two archetype organic electronic materials. While long range order has been demonstrated at the monolayer level for a number of organic systems, the thick quasi-epitaxial films (hundreds of Ångstroms) demonstrated here have practical potential for thin film organic electronic devices. Additionally, we have shown the relationship between film surface morphology and crystalline ordering, and demonstrated that a flat surface that accompanies long range film order can be achieved at low growth temperatures on templating substrates.

\section{Experimental}

Growth was carried out in a vertical, multibarrel quartz OVPD chamber described previously [26]. Crystal structure was monitored in situ and in real-time with HP-RHEED [24], and ex situ with X-ray diffraction in the Bragg-Brentano configuration using a Rigaku $\mathrm{Cu} \mathrm{K \alpha}$ rotating anode source. HP-RHEED patterns, using a $0.1 \times 20 \mathrm{~mm}^{2}$ electron beam, were recorded at a beam energy, current, and incident angle of $20 \mathrm{keV},<100 \mathrm{nA}$, and $\sim 1^{\circ}$, respectively. Beam current was minimized to avoid charging at $>100 \mathrm{nA}$. Film thickness was measured post-growth using a variable-angle spectroscopic ellipsometer on solvent-cleaned $\mathrm{Si}$ substrates. Surface topography was observed using AFM and SEM after coating the surface with $20 \AA$ of Au to prevent charging in the SEM. AFM images were used to obtain rootmean-square roughness information as well as average feature heights (see figure captions).

Both PTCDA and CuPc were twice purified by gradient sublimation prior to loading into quartz source boats located in separate barrels in the OVPD chamber [27]. CuPc was evaporated at source temperatures of $405^{\circ} \mathrm{C}$ and $415^{\circ} \mathrm{C}$ at a $\mathrm{N}_{2}$ carrier gas flow rate of $25 \mathrm{sccm}$ corresponding to nominal deposition rates of $0.4 \AA \mathrm{s}^{-1}$ and $1.2 \AA \mathrm{s}^{-1}$, respectively, at a growth pressure of 10 mTorr. PTCDA was evaporated at $385^{\circ} \mathrm{C}$ at a $25 \mathrm{sccm} \mathrm{N}$ flow, corresponding to a nominal deposition rate of $0.7 \AA \mathrm{s}^{-1}$ at a growth pressure of $60 \mathrm{mTorr}$. Single crystal $\mathrm{KBr}$ substrates were cleaved immediately prior to being loaded into the growth chamber.

To index the HP-RHEED patterns, the $d$-spacings were calculated for each assigned streak location, calibrated using the $\mathrm{KBr}$ pattern. $\mathrm{X}$-ray diffraction was used to determine the stacking direction of the film to help identify in-plane surface meshes. Lattice constants were fit to the $d$-spacings, and assigned indices using a non-linear least squares regression of all the data.

Received: July 2, 2007

Revised: July 28, 2007

Published online: November 12, 2007

[1] S. R. Forrest, Nature 2004, 428, 911.

[2] V. Podzorov, E. Menard, A. Borissov, V. Kiryukhin, J. A. Rogers, M. E. Gershenson, Phys. Rev. Lett. 2004, 93.

[3] H. Moon, R. Zeis, E. J. Borkent, C. Besnard, A. J. Lovinger, T. Siegrist, C. Kloc, Z. N. Bao, J. Am. Chem. Soc. 2004, 126, 15322.

[4] R. Zeis, C. Besnard, T. Siegrist, C. Schlockermann, X. L. Chi, C. Kloc, Chem. Mater. 2006, 18, 244.

[5] A. L. Briseno, S. C. B. Mannsfeld, M. M. Ling, S. H. Liu, R. J. Tseng, C. Reese, M. E. Roberts, Y. Yang, F. Wudl, Z. N. Bao, Nature 2006, 444, 913.

[6] S. R. Forrest, Chem. Rev. 1997, 97, 1793.

[7] G. Witte, C. Woll, J. Mater. Res. 2004, 19, 1889.

[8] A number of terms including incommensurate epitaxy, point-on-line epitaxy, coincident epitaxy, and quasi-epitaxy have been used to describe ordered organic film growth, where the relationship between the substrate and incommensurate adlayer lattices involves rotational relationships determined by energetic minima in the soft van der Waals interactions. Here, quasi-epitaxy will be used to describe growth lacking short-range commensurability that exhibits a distinct orientational alignment between substrate and film lattices. (see ref. [6]).

[9] L. Kilian, E. Umbach, M. Sokolowski, Surf. Sci. 2004, 573, 359.

[10] J. Y. Grand, T. Kunstmann, D. Hoffmann, A. Haas, M. Dietsche, J. Seifritz, R. Moller, Surf. Sci. 1996, 366, 403. 
[11] T. Schmitz-Hubsch, T. Fritz, F. Sellam, R. Staub, K. Leo, Phys. Rev. B 1997, 55, 7972.

[12] S. R. Forrest, P. E. Burrows, E. I. Haskal, F. F. So, Phys. Rev. B 1994, 49, 11309

[13] D. E. Hooks, T. Fritz, M. D. Ward, Adv. Mater. 2001, 13, 227.

[14] C. Ludwig, R. Strohmaier, J. Petersen, B. Gompf, W. Eisenmenger, $J$. Vac. Sci. Technol. B 1994, 12, 1963.

[15] M. Ashida, Bull. Chem. Soc. Jpn. 1966, 39, 2632.

[16] A. Hoshino, Y. Takenaka, M. Izukashi, H. Yoshida, H. Miyaji, Jpn. J. Appl. Phys. Part 1 2004, 43, 4344.

[17] M. Mobus, N. Karl, T. Kobayashi, J. Cryst. Growth 1992, 116, 495.

[18] T. Schmitz-Hubsch, F. Sellam, R. Staub, M. Torker, T. Fritz, C. Kubel, K. Mullen, K. Leo, Surf. Sci. 2000, 445, 358.

[19] M. L. Anderson, V. S. Williams, T. J. Schuerlein, G. E. Collins, C. D. England, L. K. Chau, P. A. Lee, K. W. Nebesny, N. R. Armstrong, Surf. Sci. 1994, 309, 551.
[20] M. Gabriel, M. Stohr, R. Moller, Appl. Phys. A 2002, 74, 303.

[21] T. Kunstmann, A. Schlarb, M. Fendrich, T. Wagner, R. Moller, R. Hoffmann, Phys. Rev. B 2005, 71 .

[22] J. A. Last, A. C. Hillier, D. E. Hooks, J. B. Maxson, M. D. Ward, Chem. Mater. 1998, 10, 422.

[23] L. Nony, R. Bennewitz, O. Pfeiffer, E. Gnecco, A. Baratoff, E. Meyer, T. Eguchi, A. Gourdon, C. Joachim, Nanotechnology 2004, 15, S91.

[24] R. R. Lunt, J. B. Benziger, S. R. Forrest, Appl. Phys. Lett. 2007, 90, 181932.

[25] M. Eremtchenko, J. A. Schaefer, F. S. Tautz, Nature 2003, 425, 602.

[26] M. Shtein, H. F. Gossenberger, J. B. Benziger, S. R. Forrest, J. Appl. Phys. 2001, 89, 1470.

[27] M. Baldo, M. Deutsch, P. Burrows, H. Gossenberger, M. Gerstenberg, V. Ban, S. Forrest, Adv. Mater. 1998, 10, 1505. 